\title{
Nível de atividade física em indivíduos com Síndrome Patelofemoral
}

\author{
Altair Argentino Pereira Júnior ${ }^{1}$ \\ Walter Celso de Lima \\ ${ }^{1,2}$ Universidade do Estado de Santa Catarina - UDESC, Florianópolis, SC, Brasil
}

\begin{abstract}
Resumo: O objetivo deste estudo foi verificar o nível de atividade física em indivíduos com Síndrome Patelofemoral (SPF). A amostra foi composta por 50 indivíduos com diagnóstico médico de SPF. Como instrumento para coleta de dados utilizou-se o Questionário Internacional de Atividade Física (IPAQ). Os dados foram analisados pela estatística descritiva. Os resultados mostraram que o dispêndio energético em atividade física no trabalho foi alto. Poucos indivíduos executaram atividades físicas como meio de transporte. As mulheres despenderam maior tempo em atividades em domésticas. A maioria dos participantes do estudo realizou alguma atividade de lazer ou exercício físico. No entanto, os mesmos apresentaram baixos níveis de atividade física, o que pode estar relacionado com a SPF, e ainda, períodos prolongados gastos na posição assentada, sendo este um fator agravante desta disfunção musculoesquelética.
\end{abstract}

Palavras-chave: Atividade física. Joelho. Síndrome da Dor Patelofemoral.

\section{Physical activity level in people with Patellofemoral Syndrome}

\begin{abstract}
The aim of this study was to verify the level of physical activity of individuals with patellofemoral syndrome (PFS). The sample was composed of 50 individuals diagnosed by their attending physicians as having PFS. Data was collected by International Physical Activity Questionnaire (IPAQ). The data was analyzed through descriptive statistics. Results showed a high waste of energy in physical activity during the exercises. Few people use physical activity as a mean of transportation. Women spend more time with activities at home. Most of the people researched practice physical exercises or have a leisure time. However, they had low levels of physical activity, which may be related PFS, and also spent long periods in the seated position, which is an aggravating factor of this musculoskeletal dysfunction.
\end{abstract}

Key Words: Physical activity. Knee. Patellofemoral Pain Syndrome.

\section{Introdução}

A articulação do joelho está envolvida em cerca de $50 \%$ das lesões músculo-esquelética. Diversas lesões podem acontecer no joelho, desde aquelas provocadas por traumas diretos, como fraturas, lesões parciais ou completas de ligamentos e meniscos, por movimentos que envolvem torção e suporte de carga. Existe também um outro grupo importante de lesões, que são as que ocorrem nos tendões em decorrência de traumas diretos, ou em virtude do uso excessivo, ou inadequado da articulação, relacionado aos aspectos biomecânicos (PEREIRA JÚNIOR e LIMA, 2008).

A Síndrome Patelofemoral (SPF), é definida como uma dor anterior ou retropatelar na articulação do joelho, sendo resultante de alterações físicas e biomecânicas na articulação patelofemoral (BAKER et al, 2002).

\footnotetext{
${ }^{1}$ Fisioterapeuta, Mestre em Ciências do Movimento Humano UDESC

${ }^{2}$ Professor do Mestrado em Ciências do Movimento Humano - UDESC. Doutor em Ciências.
}

A dor na articulação patelofemoral é um dos sintomas comum que afeta todas as faixas etárias. Vinte e cinco por cento da população irá sofrer com sintomas patelofemorais em algum momento da vida (SERVI, 2008).

$\mathrm{Na}$ população em geral, a síndrome patelofemoral afeta mais as mulheres do que os homens, em uma proporção de 2:1 (SERVI, 2008). Baker et al (2002), relataram que esta condição musculoesquelética dolorosa afeta principalmente adolescentes e jovens fisicamente ativos. Já entre os atletas a prevalência da síndrome patelofemoral é maior no sexo masculino (MALANGA e LEE, 2008). De acordo com Dugan (2005), a síndrome patelofemoral afeta em maior porcentagem as mulheres atletas do que os homens atletas quando estes executam a mesma atividade física, e o mesmo nível de competição.

Os dados sobre a prevalência de atividade física (AF) na população mundial são escassos. A literatura relata à associação entre a prática de $A F$, saúde e qualidade de vida, apresentando uma relação direta entre o nível de AF e aumento 
da longevidade, o que incentiva as pessoas a praticar AF (PARDINI et al, 2003).

O sedentarismo pode gerar disfunções músculo-esqueléticas, e desequilíbrio entre as forças musculares exercidas na articulação do joelho, gerando desalinhamento articular e hiperpressão patelar. Um mau alinhamento atribuído a forças de pressão aplicadas pelo quadríceps e tendão patelar, gera uma distribuição anormal da força de pressão na articulação patelofemoral, o que, por conseguinte gera dor e degeneração articular (ELIAS et al, 2004).

A recomendação de AF feita pelo Centro de Controle e Prevenção de Doenças dos Estados Unidos e pelo Colégio Americano de Medicina Esportiva, é que todo adulto deve realizar trinta minutos ou mais de atividade física de moderada intensidade, preferencialmente todos os dias da semana (LEE e PAFFENBARGER, 1996).

Sendo assim, mensurar o nível de AF é cada vez mais importante no contexto da saúde pública. Entretanto a literatura apresenta vários métodos para medir este parâmetro, o que dificulta a comparação dos resultados e padronização de um método específico para mensurar o nível de atividade física (HALLAL, 2003).

Uma das formas de mensurar o nível de AF é através do IPAQ que permite estimar o dispêndio energético semanal de atividades físicas relacionadas com o trabalho, transporte, tarefas domésticas, lazer e tempo sentado, com intensidade vigorosa, moderada, leve, realizadas por pelo menos 10 minutos contínuos

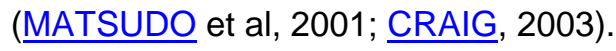

Devido o IPAQ investigar sobre as atividades físicas executadas em diferentes condições ambientais ele se torna útil para ser aplicado em indivíduos com síndrome patelofemoral, uma vez que, é possível estabelecer em que condição o indivíduo é mais ativo ou pouco ativo.

Através da aplicação do IPAQ em indivíduos com SPF pretendeu-se obter dados que possam colaborar na avaliação e tratamento dos indivíduos com SPF. Dessa forma o objetivo deste estudo foi verificar o nível de atividade física em indivíduos com Síndrome Patelofemoral (SPF).

\section{Metodologia}

A amostra foi composta por 50 voluntários $(39,96 \pm 14,81$ anos) sendo 33 mulheres (66\%) $(37,84 \pm 14,42$ anos) e 17 homens (34\%) com média de idade $(44,17 \pm 14,79$ anos). Todos os participantes da pesquisa tinham o diagnóstico médico de SPF. Foram excluídos da amostra indivíduos que apresentassem outras lesões na região do joelho ou que comprometesse o entendimento das atividades solicitadas.

A pesquisa teve a aprovação do comitê de ética em pesquisa da Universidade do Estado de Santa Catarina (UDESC) com o parecer número 121/05.

Para verificar a prática de AF nos voluntários, foi utilizado o Questionário Internacional de Atividade Física (IPAQ), versão longa.

A pesquisa foi realizada na clínica de Fisioterapia Fisiocentro, localizada na cidade de Balneário Camboriu, estado de Santa Catarina. O questionário utilizado no estudo foi aplicado na forma de entrevista por um único pesquisador. Foi explicada aos participantes, a importância da pesquisa e solicitado a assinatura do termo de consentimento livre e esclarecido em 2 vias.

Os resultados obtidos foram categorizados de acordo com o instrumento usado para coleta. Fez-se uso da estatística descritiva e do Software Microsoft Excel 2003, para elaborar as planilhas e gráficos.

\section{Resultados}

Serão apresentados os resultados obtidos através do Questionário Internacional de Atividade Física (IPAQ), em seus diferentes domínios (AF no trabalho, transporte, atividades domésticas, exercício e lazer), classificando a amostra em 2 níveis: não ativos (< $150 \mathrm{~min} / \mathrm{sem}$ ) e ativos (> $150 \mathrm{~min} / \mathrm{sem}$ ).

No domínio I do IPAQ (AF no trabalho), 58,8\% dos homens foram classificados fisicamente como não ativos, pois não realizaram no mínimo 10 minutos contínuos de AF. As mulheres classificadas como não ativas (69,7\%) executaram em média (11,74 \pm 32,84 min/sem). O total de indivíduos não ativos foi de $66 \%$ da amostra com nível de AF executado de $(8,18 \pm$ $27,77 \mathrm{~min} / \mathrm{sem})$. Os homens classificados como ativos (41,2\%) executaram em média (1967 \pm $1294,75 \mathrm{~min} / \mathrm{sem}$ ) de AF durante o trabalho. Já as mulheres consideradas ativas (30,3\%) executaram $(1652 \pm 1312,70 \mathrm{~min} / \mathrm{sem}$ durante 0 trabalho). Do total de indivíduos ativos da amostra 34\%, o nível de AF no trabalho durante a semana foi de $(1781,8 \pm 1274,15 \mathrm{~min} / \mathrm{sem})$.

No domínio II do IPAQ, AF no meio de transporte, que se relaciona com o deslocamento de um lugar para o outro, através de caminhada ou bicicleta, observou-se que $64,7 \%$ dos homens gastaram 25,45 $\pm 54,16 \mathrm{~min} / \mathrm{sem}$ neste tipo de 
AF, o que classifica estes indivíduos como não ativos. Já as mulheres não ativas $69,7 \%$ executaram $38,48 \pm 57,47 \mathrm{~min} / \mathrm{sem}$ em seus deslocamentos. Sessenta e oito por cento dos indivíduos participantes da pesquisa foram classificados como não ativos com uma média de AF de $30,73 \pm 55,91 \mathrm{~min} / \mathrm{sem}$. Os homens classificados como fisicamente ativos (35,3\%) executaram 295, $83 \pm 89,91 \mathrm{~min} / \mathrm{sem}$ de AF como meio de transporte. Entre as mulheres (30,3\%) a média de AF foi de $402 \pm 470,47 \mathrm{~min} / \mathrm{sem}$. Assim o total de indivíduos fisicamente ativos nesse domínio foi de $32 \%$ executando semanalmente $362,18 \pm 371,91 \mathrm{~min} / \mathrm{sem}$ de AF.

No domínio III do IPAQ (AF em casa), incluiu atividades executadas durante uma semana dentro e ao redor do domicílio. Neste domínio, $82,3 \%$ dos homens foram classificados como não ativos $(6,42 \pm 24,05 \mathrm{~min} / \mathrm{sem})$ e as mulheres classificadas como não ativas foram $48,5 \%$ $(39,37 \pm 55,19 \mathrm{~min} / \mathrm{sem})$. Ao analisar o total de indivíduos não ativos da amostra (60\%) o tempo gasto com AF foi de $24 \pm 45,98 \mathrm{~min} / \mathrm{sem}$. Os homens classificados como fisicamente ativos
$(17,7 \%)$ executaram $610 \pm 170,58 \mathrm{~min} / \mathrm{sem}$. Entre as mulheres ativas $(51,5 \%)$ o nível de AF em casa foi de $923,52 \pm 817,14 \mathrm{~min} / \mathrm{sem}$. Dessa forma o total de indivíduos ativos (40\%) gastaram $876,50 \pm 760,62 \mathrm{~min} / \mathrm{sem}$ em AF dentro ou ao redor da habitação.

O domínio IV do IPAQ se refere às atividades físicas de recreação, esportes, exercício e lazer que os indivíduos executam durante uma semana normal. Neste domínio, $41,2 \%$ dos homens classificados como não ativos executaram em média $79,29 \pm 46,40 \mathrm{~min} / \mathrm{sem}$ e as mulheres consideradas não ativas $(51,5 \%)$ despenderam $23,24 \pm 48,89 \mathrm{~min} / \mathrm{sem}$ de AF. O total de indivíduos não ativos neste domínio foi de $48 \%$ com um tempo médio gasto com AF de 39,59 \pm $53,87 \mathrm{~min} / \mathrm{sem}$. Os homens ativos $(58,8 \%)$ executaram $372,50 \pm 227,66 \mathrm{~min} / \mathrm{sem}$ de $\mathrm{AF}$ neste domínio. Entre as mulheres ativas (48,5\%) o dispêndio foi de 550,94 $\pm 464,04 \mathrm{~min} / \mathrm{sem}$. O total de indivíduos ativos neste domínio correspondeu a $52 \%$ da amostra que executaram $482,30 \pm 394,58 \mathrm{~min} / \mathrm{sem}$ de AF.

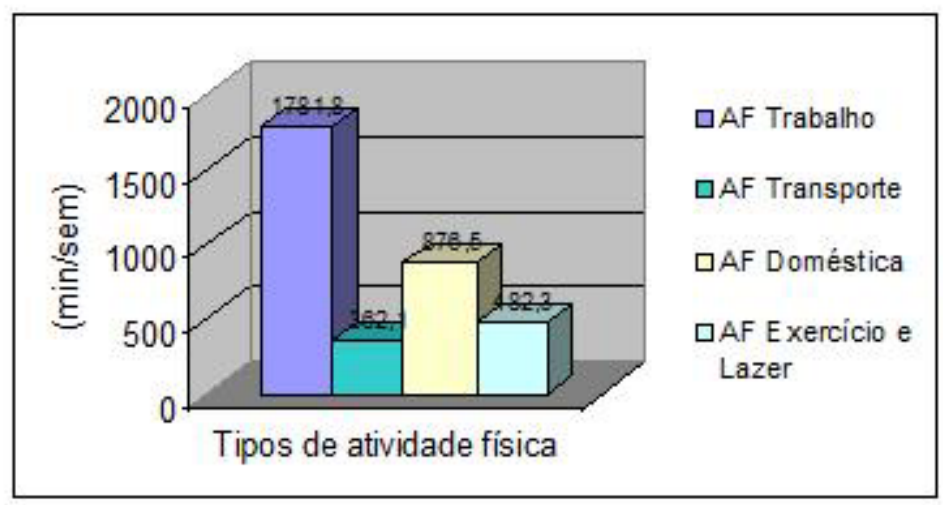

Figura 1. Questionário Internacional de Atividade Física (IPAQ). Contribuição das diferentes formas de (AF) em minutos por semana.

Os dados apresentados na figura 1 mostram a média de minutos que os indivíduos classificados como ativo, executaram nos diferentes domínios. O dispêndio com AF no trabalho foi de 1781,8 \pm $1274,15 \mathrm{~min} / \mathrm{sem}$, no meio de transporte $362,18 \pm$ $371,91 \mathrm{~min} / \mathrm{sem}$, AF doméstica 876,50 $\pm 760,62$ $\mathrm{min} / \mathrm{sem}$ e a, AF no domínio exercício e lazer foi de 482,30 $\pm 394,58 \mathrm{~min} / \mathrm{sem}$.

Através do IPAQ foi verificado o tempo que os participantes ficaram assentados a semana, no final de semana, e no transporte em ônibus, carros ou outros veículos. Todos os indivíduos do estudo despenderam tempo sentado.

Entre os homens o tempo gasto sentado durante os dias da semana foi de $1100 \pm 835,35$ $\mathrm{min} / \mathrm{sem}$, no final de semana foi de $471,76 \pm$ $290,43 \mathrm{~min} / \mathrm{sem}$, no meio de transporte foi de $772,35 \pm 915,85 \mathrm{~min} / \mathrm{sem}$ perfazendo um total de tempo gasto de $2344,11^{\prime} \pm 1146,79 \mathrm{~min} / \mathrm{sem}$. As mulheres gastaram em média $(1336,36 \pm 848$ $\mathrm{min} / \mathrm{sem}$ ) sentadas durante os dias da semana. No final de semana o tempo gasto na posição sentada foi de $574,54 \pm 360,94 \mathrm{~min} / \mathrm{sem}$. No transporte, as mulheres despenderam 304,85 \pm $351,07 \mathrm{~min} / \mathrm{sem}$ perfazendo um total de tempo gasto sentada durante uma semana de 2215,75' $\pm 1176,38 \mathrm{~min} / \mathrm{sem}$. Ao se observar a amostra como um todo, independente dos gêneros, o tempo gasto sentado durante os dias da semana foi de $1256 \pm 842 \mathrm{~min} / \mathrm{sem}$, no final de semana $539,60 \pm 339,18 \mathrm{~min} / \mathrm{sem}$, no meio de transporte 
foi de $463,80 \pm 635,94 \mathrm{~min} / \mathrm{sem}$, perfazendo um total de $2259,4^{\prime} \pm 1159,20 \mathrm{~min} / \mathrm{sem}^{\prime}$, na posição assentada durante os 7 dias da semana.

\section{Discussão}

Os resultados mostraram que os homens gastaram $2344,11 \pm 1146,79 \mathrm{~min} / \mathrm{sem}$ na posição sentada, e mulheres tiveram um tempo de permanência assentada de 2215,75 $\pm 1176,38$ $\mathrm{min} / \mathrm{sem}$. Observa-se que o tempo gasto sentado por homens foi maior, devido os mesmos despenderem mais tempo sentados no meio de transporte. Como citado anteriormente, períodos prolongados de permanência na posição sentada parecem ser uma das causas da SPF (PEREIRA JÚNIOR e LIMA, 2008). Forças de pressão aplicadas pelo quadríceps e tendão patelar geram uma distribuição anormal da força de pressão na articulação patelofemoral, com desenvolvimento de dor e degeneração articular (WOOD et al, 2008; ELIAS et al, 2004).

O exercício físico tornou-se uma unanimidade na promoção da saúde e melhora da qualidade de vida, diminuindo o risco de doenças crônicas e atuando como fator chave para aumentar a longevidade. Como conseqüência do controle do sedentarismo através da prática regular de exercícios, da mudança do padrão estético e das exigências do esporte competitivo é comum atletas e não atletas, em todos os níveis de performance, excederem os limites de sua capacidade física e psicológica (UUSITALO, 2001).

Atualmente os indivíduos têm sido estimulados a praticar atividade física. Sendo a atividade física um dos fatores que podem contribuir para o desencadeamento da SPF, quando a AF não é executada de forma adequada, ou por falta de preparo dos praticantes e ainda pelo excesso de treinamento (PEREIRA e LIMA, 2007).

As atividades de vida diária e atividades físicas executadas de maneira incorreta, posturas inadequadas, são fatores que podem favorecer 0 surgimento da SPF (BAKER et al, 2002).

Nesse estudo, a atividade física realizada no trabalho, demonstrou que $66 \%$ dos participantes executaram atividades laborais com pouco dispêndio energético, não atingindo 10 minutos de AF diárias como parte do trabalho, isso significa que, grande parte destes indivíduos executaram seu trabalho de forma sentada. Estudos realizados por Linschoten et al (2006), observaram que na maioria das vezes os pacientes com SPF relatam uma dor na parte anterior do joelho, na região da patela e dizem que esta dor piora depois de terem ficado sentados por muito tempo (sinal do cinema) o que corrobora com os nossos dados onde foi observado longos períodos gastos na posição sentada entre os participantes.

Entre os indivíduos classificados como ativos observa-se um nível de AF durante o trabalho bastante alto $(1781,8 \pm 1274,15 \mathrm{~min} / \mathrm{sem})$. Esses indivíduos executaram atividades laborais que envolveram caminhadas como parte do trabalho ou movimentos que necessitavam de força para execução de seu trabalho, o que gera grande gasto energético. A execução de atividades físicas sem preparo prévio como aquecimento e alongamento, pode ocasionar diferentes tipos de lesões no sistema músculo-esquelético, principalmente se associado ao uso excessivo ou inadequado da articulação (PEREIRA JÚNIOR e LIMA, 2008).

Ao se investigar as AF executadas como parte do trabalho observa-se que a maior parte dos indivíduos exerceu atividades com pouco dispêndio energético, ficando principalmente sentados, visto que esta é uma postura que afeta a articulação do joelho e favorece o desencadeamento da SPF (ELIAS et al, 2004). Por outro lado os indivíduos classificados como fisicamente ativos no trabalho, exerceram níveis altos de AF durante a semana. Supõe-se então que estas AF foram executadas sem preparo prévio, o que gera uso excessivo e inadequado da articulação, com possibilidade de desenvolvimento da SPF.

A atividade física como meio de transporte está relacionada com a maneira que os indivíduos se deslocam no seu dia a dia. Neste estudo observou-se que $68 \%$ dos indivíduos foram classificados como não ativos no meio de transporte, gastaram em média, 30,73 \pm 89,91 $\mathrm{min} / \mathrm{sem}$ em seus deslocamentos. A grande maioria usou carro ou ônibus como meio de transporte. Apenas $32 \%$ dos participantes deslocaram de maneira que gerava dispêndio energético (362,18 $\pm 371,91 \mathrm{~min} / \mathrm{sem})$.

As atividades físicas executadas em casa (dentro e ao redor da habitação), classificaram $51,5 \%$ das mulheres como ativas. Estas voluntárias executaram 923, $52 \pm 817,14 \mathrm{~min} / \mathrm{sem}$ de atividades relacionadas com tarefas domésticas, de limpeza no interior da casa e cuidados com o jardim. Hallal et al (2003), relataram que o IPAQ é um dos poucos instrumentos que coleta informações sobre AF executadas no domicílio. Na maioria das vezes este tipo de AF é executado por mulheres. Ao investigar o nível de AF em mulheres idosas, 
Mazo et al (2005), destacaram que a maioria das idosas executam AF com uma intensidade moderada dentro e ao redor da habitação. Entre os homens classificados como ativos neste estudo (17,7\%), a média de AF executada em casa foi de $610 \pm 170,58 \mathrm{~min} / \mathrm{sem}$ o que demonstrou que os homens são menos ativos que as mulheres neste domínio.

No presente estudo as atividades físicas de recreação, esporte, exercício e lazer, classificaram $58,8 \%$ dos homens como ativos, com $372,50 \pm 227,66 \mathrm{~min} / \mathrm{sem}$ gastos com AF neste domínio. Entre as mulheres consideradas ativas $(48,5 \%)$ o dispêndio com AF foi de 550,94 $\pm 464,04 \mathrm{~min} / \mathrm{sem}$. Observa-se que a prática de AF neste domínio é próxima entre homens e mulheres, porém as mulheres despendem maior tempo por semana na execução de atividade física nesse domínio. Destaca-se ainda que este foi o domínio que classificou a maior parte da amostra como ativos, correspondendo a $52 \%$ dos participantes.

No estudo realizado por Levinger e Gilleard (2005), com finalidade de investigar a marcha em indivíduos com SPF e indivíduos assintomáticos, os autores constataram que os participantes de ambos os grupos do estudo eram fisicamente ativos, participavam de atividades esportivas recreacionais durante a semana, totalizando 3,2 horas (200 minutos) nos indivíduos com SPF e 4,1 horas (250 minutos) no grupo assintomático. No presente estudo foi observado que os participantes executaram 482,30 \pm 394,58 $\mathrm{min} / \mathrm{sem}$ de AF esportivas ou de lazer. De acordo com Bevilaqua-Grossi et al (2008), as respostas reflexas dos músculos estabilizadores patelares de indivíduos assintomáticos e com SPF não apresentam diferenças significativas.

Em relação à inatividade física, $18,2 \%$ dos homens e $22,6 \%$ das mulheres não realizavam nenhum tipo de AF esportiva. A prevalência de inatividade física tem sido demonstrada em alguns estudos (HALLAL et al, 2003; MONTEIRO et al, 2003). Hallal et al (2003), observaram prevalência de inatividade física de $41,1 \%$ para a faixa etária de 20 a 65 anos, verificado através do IPAQ. Em comparação com os estudos citados acima, 22\% da amostra desse estudo, o que equivale a 11 participantes apresentou índices de sedentarismo relacionado à prática de $\mathrm{AF}$ esportiva. É sabido que o sedentarismo é uma condição indesejável e representa riscos para a saúde, gerando diferentes doenças crônicodegenerativas e alterações no sistema osteomioarticular, entre estas a SPF (WOOD et al, 2008).
Entretanto, uma limitação do nosso estudo foi que a amostra estudada era somente sintomática. Dessa forma outros estudos clínicos controlados e aleatorizados devem ser realizados com a finalidade de obterem-se dados para verificar diferenças no nível de atividade física praticado por indivíduos com e sem SPF.

\section{Conclusões}

Conclui-se que o nível de AF em indivíduos com SPF foi baixo, o que pode ser ocasionado pela alteração funcional do joelho, dificultando a prática da AF. O único domínio que classificou a maior parte da amostra como fisicamente ativos, foi à prática de AF de recreação, esporte, exercício e lazer.

O tempo gasto na posição sentada da nossa amostra foi bastante alto, o que pode contribuir para o desencadeamento da SPF, devido aumento da pressão patelar.

Através desta pesquisa observou-se que 0 IPAQ é um bom instrumento para mensurar o nível de AF, pois, com a utilização deste, investigaram-se os diferentes meios em que 0 indivíduo com SPF, vive e executa os diferentes tipos de AF, conhecendo assim onde o indivíduo é mais ativo ou insuficientemente ativo, dados estes que contribuem para a avaliação e melhor entendimento dos indivíduos com SPF.

\section{Referências}

BAKER, V. et al. Abnormal knee position sense in individuals with patellofemoral pain syndrome.

Journal of Orthopaedic Research, v.20, 2002. http://dx.doi.org/10.1016/S0736-0266(01)00106-1

BEVILAQUA-GROSSI, D. FELÍCIO, L.R.

LEOCÁCIO, L.P. Análise do tempo de resposta reflexa dos músculos estabilizadores patelares em indivíduos com síndrome da dor patelofemural. Revista Brasileira de Fisioterapia, v.11, n.5, 2008.

CRAIG, C.L. et al. International physical activity questionnarie: 12-country realiabiliry and validiry. Medicine \& Science in Sports \& Exercise, v.35, n.8, 2003.

DUGAN, S.A. Sports-related knee injuries in female athletes: What gives? American Journal of Physical Medicine \& Rehabilitation, v.84, n.2, 2005.

ELIAS, J.J.; WILSON, D.R.; ADAMSON, R.; COSGAREA, A.J. Evaluation of a computational model used to predict the patellofemoral contact pressure distribution. Journal of Biomechanics, v.37, 2004. 
HALLAL, P.C. et al. Physical inactivity: Prevalence and associated variables in brazilian adults. Medicine \& Science in Sports \& Exercise, v.35, n.11, 2003.

LEE, I. PAFFENBARGER JR, R.S. How much physical activity is optimal for health? Methodological considerations. Research Quarterly for Exercise and Sport, v.67 n.2, 1996.

LEVINGER, P.; GILLEARD, W. The heel strike transient during walking in subjects with patellofemoral pain syndrome. Physical Therapy in Sport, v.20, 2005.

http://dx.doi.org/10.1016/i.ptsp.2005.02.005

LINSCHOTEN, R.V. et al. The PEX study Exercise therapy for patellofemoral pain syndrome: design of a randomized clinical trial in general practice and sports medicine. BMC musculoskeletal disordens, v.7, n.31, 2006. http://dx.doi.org/10.1186/1471-2474-7-31

MALANKA, G.A. LEE, W.S. Patellar Injury and dislocation. Set. 2008. Disponível em: <http:// emedicine.medscape.com/articles/90068> Acesso em 02 fev. 2009.

MATSUDO S. et al. Questionário Internacional de Atividade Física (IPAQ): Estudo da Validade e Reprodutibilidade no Brasil. Revista Brasileira de Atividade Física e Saúde, v.6, n.2, 2001.

MAZO, G.Z. et al; Nível de atividade física, condições de saúde e características sóciodemográficas de mulheres idosas brasileiras. Revista Portuguesa de Ciências Desportiva, v.2, 2005.

MONTEIRO, C.A. et al; A descriptive epidemiology of leisure-time physical activity in Brazil, 1996-1997. Revista Panamericana Salud Publica/Pan Am/ Public Health, v.14, n.4, 2003.

PARDINI R. et al. Validação do questionário internacional de nível de atividade física (IPAQ versão 6): estudo piloto em adultos jovens brasileiros. Revista Brasileira de Ciência e Movimento, v.9, n.3, 2003.

PEREIRA JÚNIOR, A.A., LIMA, W.C. Avaliação do nível e tipo de atividade física em individuos com Síndrome Patelofemoral. Revista da UNIFEBE. n. 5, 2007.

PEREIRA JÚNIOR, A.A.; LIMA, W.C. Relação entre função do joelho e índice de massa corporal na síndrome patelofemoral. Revista Terapia Manual, v.6, n.26, 2008.

SERVI, J.T. Patellofemoral joint syndromes. Mar. 2008. Disponível em:
$<$ http://emedicine.medscape.com/articles/90286> acesso 18 fev 2009.

UUSITALO, A.L.T. Overtraining. Making a difficult diagnosis and implementing targeted treatment. The Physician and Sport Medicine, v.29, n.5, 2001.

WOOD, L. PEAT, G. THOMAS, L. HAY, E.M. SIM, J. Associations between physical examination and self-reported physical function in older community-dwelling adults with knee pain. Physical Therapy, v. 88, n.1, 2008. http://dx.doi.org/10.2522/ptj.20060372

Endereço:

Altair Argentino Pereira Júnior Rua 3150, n 35 apto 403 Centro Balneário Camboriú SC Brasil 88330-281

Telefone: (47) 3368-5667 (47) 9931-3123

e-mail: altjunior@unifebe.edu.br

Recebido em: 18 de maio de 2009.

Aceito em: 22 de novembro de 2009.

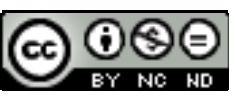

Motriz. Revista de Educação Física. UNESP, Rio Claro, SP, Brasil - elSSN: 1980-6574 - está licenciada sob Licença Creative Commons 